\title{
Gastrointestinal Bleed from a Left Ventricle to Colonic Interposition Graft Fistula following an Esophagectomy
}

\author{
Abdulwahab Al Khalifa ${ }^{1}$ William Gourdin McMaster, Jr. ${ }^{2}$ Colin Schieman ${ }^{3}$ Richard Whitlock ${ }^{4}$ \\ Christopher Ricci ${ }^{5}$ Matthew Danter ${ }^{6}$ \\ ${ }^{1}$ Division of Cardiac Surgery, The University of Toronto, Toronto, Canada \\ 2 Thoracic Surgery Resident, Vanderbilt University Medical Center, \\ Nashville, TN \\ ${ }^{3}$ Division of Thoracic and Esophageal Surgery, McMaster University, \\ Hamilton, Ontario, Canada \\ ${ }^{4}$ Division of Cardiac Surgery, McMaster University, Hamilton, Ontario, \\ Canada \\ ${ }^{5}$ Department of Anesthesia, McMaster University, Hamilton, Ontario, \\ Canada \\ ${ }^{6}$ Department of Cardiac Surgery, Vanderbilt University Medical \\ Center, Nashville, TN \\ Thorac Cardiovasc Surg Rep 2017;6:e22-e24. \\ Address for correspondence William Gourdin McMaster, Jr., MD, \\ Vanderbilt University Medical Center, 1161 21st Ave S, CCC-4312 \\ MCN, Nashville, TN 37232-2102, United States \\ (e-mail: william.g.mcmaster@vanderbilt.edu).
}

\begin{abstract}
Keywords

- left ventricle

- colonic interposition graft

- gastrointestinal bleed

Colonic interposition grafts are commonly used as an esophageal conduit following esophageal resection. Significant morbidity is associated with this reconstruction due to the nature of the operation. Many of the complications associated with this procedure have clear management strategies; however, there is a paucity of data when it comes to managing rare complications. In this report, we discuss the presentation, operative intervention, and postoperative care of a patient who presented with a left ventricle to esophageal colonic interposition graft fistula.
\end{abstract}

\section{Introduction}

The use of colonic interposition grafts to reconstruct the thoracic alimentary canal is an established technique for many esophageal conditions. Contemporary mortality rates for this type of esophageal reconstruction are between 5 and $8 \%$ with morbidity rates between 55.7 and $65 \%{ }^{1,2}$ Common complications include pneumonia, arrhythmias, wound infections, anastomotic leaks, graft ischemia, and stricture. ${ }^{1,3}$ Cardiac complications are extremely rare with only one case being reported in the literature. ${ }^{4}$

\section{Case Description}

A 46-year-old female presented to the emergency department with an exacerbation of chronic left-sided chest pain and a several month history of malaise, fatigue, and weight loss. The patient's surgical history was significant for esophageal reconstruction using a colonic interposition graft for esophageal atresia, a colonic interposition graft revision, and

received

February 27, 2017

accepted after revision

April 24, 2017 a strictureplasty at the cologastric junction. During her hospital course, she developed hematemesis, hematochezia, and hypotension. Computed tomography (CT) of the chest demonstrated extravasation of contrast from the left ventricle (LV) into the colonic interposition graft $(-$ Fig. 1A). The patient was transferred to the cardiac surgery service with the diagnosis of a left ventricular-colonic interposition graft fistula. Transthoracic echocardiography demonstrated a left ventricular pseudoaneurysm (-Fig. 1B), normal left ventricular function, mild-to-moderate mitral regurgitation, and no evidence of endocarditis. Doppler echocardiography demonstrated flow within the pseudoaneurysm ( - Fig. 1C), and angiography confirmed a left ventricular pseudoaneurysm (-Fig. 1D) without significant coronary artery disease.

The patient was taken to the operating room emergently. Peripheral cannulation of the left femoral artery and right femoral vein for cardiopulmonary bypass was performed. Median sternotomy and pericardial adhesiolysis were performed without incident. The left ventricular apex was adhered to the pericardium posteriorly. Cardiopulmonary bypass was

\footnotetext{
(c) 2017 Georg Thieme Verlag KG Stuttgart · New York
}

License terms

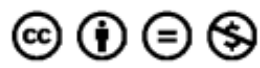


A
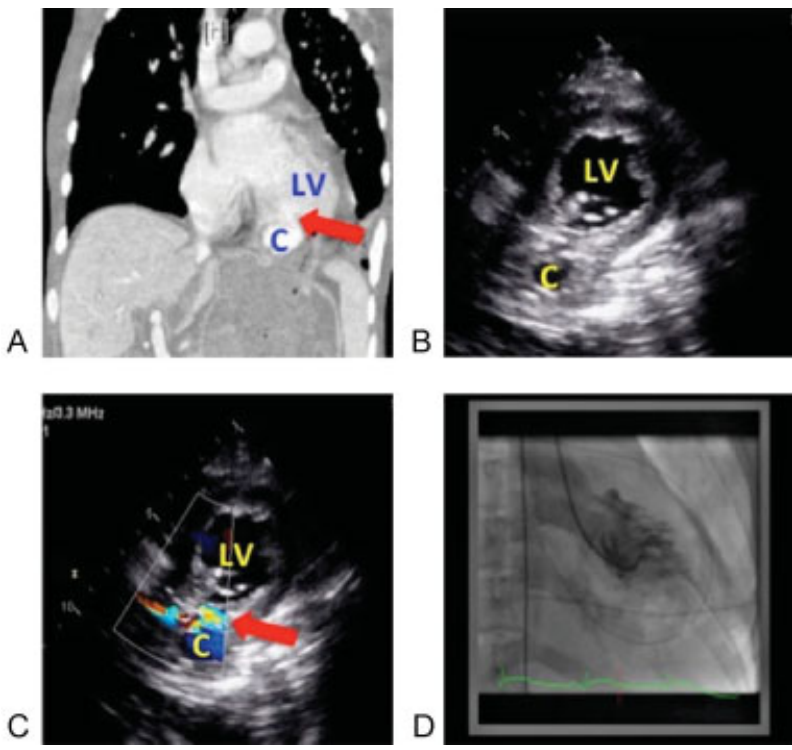

Fig. 1 (A) Computed tomography (CT) angiography. (B) Transthoracic echocardiography: parasternal short axis. (C) Transthoracic color Doppler echocardiography: parasternal short axis. (D) Angiography. C, Colon; LV, left ventricle; red arrow, ventriculocolonic fistula.

initiated and dissection toward the left ventricular apex continued. Diastolic cardioplegic arrest was achieved, and the mature fistula tract was mobilized circumferentially. The fistula was entered at the level of the pericardial reflection, and a significant amount of clot and fibrosis were encountered in the communicating pericardial-colonic graft defect (-Fig. 2A, B). Once incised, the fibrosed area measured ap-
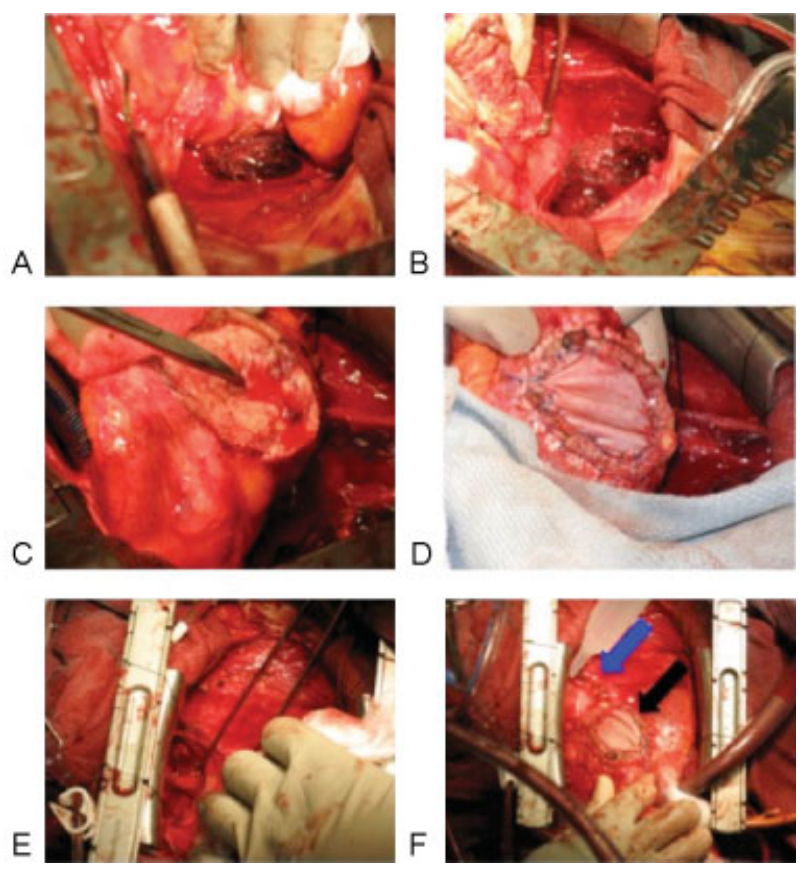

Fig. 2 (A) Entering the ventriculocolonic fistula tract. (B) Clot in the pericardial defect. (C) Actively bleeding ventriculocolonic fistula. (D) Repair of ventriculocolonic fistula. (E) Pericardial defect penetrating into the colonic interposition graft. (F) Repair of left ventricle (black arrow) and the pericardial-colonic defect (blue arrow). proximately $9 \times 6 \mathrm{~cm}$. There was a $1.5-\mathrm{cm}$ defect with active extravasation, which was bordered by dense fibrous tissue, on the LV ( - Fig. 2C). A bovine pericardial patch was used to close the left ventricular fistula. The patch was secured with a double layer of 3-0 polypropylene sutures: the inner layer consisted of pledgeted, interrupted sutures, and the outer layer was a running suture (-Fig. 2D). Fibrin sealant was applied as an adjunct to hemostasis. The colonic wall and the pericardium were fused (-Fig. 2E). Given the patient's tenuous hemodynamics and the extensive surgery required for definitive repair, the decision was made to exclude the pericardium using an autologous pericardial patch (-Fig. 2F). The area was extensively drained with a nasogastric tube, and three thoracostomy tubes were placed: in the pericardium, in the anterior mediastinum, and in the right contralateral chest. Hemostasis was achieved; the patient was successfully weaned from cardiopulmonary bypass and was transferred to the cardiac intensive care unit in critical but stable condition.

On postoperative day 4 , the thoracic surgery team took the patient to the operating room for definitive colonic repair. The colonic interposition graft was mobilized through a left thoracotomy. The defect was noted to extend into the cologastric junction. In an attempt to preserve the interposition graft, the defect was primarily closed, and the cologastric junction was reinforced with an intercostal muscle flap. The area was drained, and the patient was returned to the intensive care unit. Postoperatively, purulent drainage was noted from the mediastinal chest drains, and the patient deteriorated clinically. Due to concern for mediastinitis, the patient was taken to the operating room for urgent reexploration. The colonic repair had broken down with drainage into the pericardium. The patient became hemodynamically unstable and definitive repair was aborted. Following 72 hours of resuscitation, she returned to the operating room and underwent resection of the colonic interposition graft, repair of the resulting gastric defect, creation of a cervical esophagostomy, and wide local drainage. After diversion, the patient did not require any further operative interventions.

Following excision of the colonic interposition graft, the patient gradually recovered and was discharged to a rehabilitation unit tolerating tube feeds and recovering well. During the postoperative period, the patient's complications included bacteremia, pneumonia, severe deconditioning, feeding tube malfunction, and nutritional challenges. Nonetheless, the patient continued to recover and was discharged home 137 days after her initial operation. Esophageal reconstruction with a long segment pedicled jejunal interposition graft may be considered when the patient has fully recovered and is nutritionally replete.

\section{Discussion}

Ventriculocolonic fistula is an extremely rare complication of a colonic interposition graft following esophagectomy. This case has instructional value as it highlights several key principles in the management of potentially catastrophic cardiac injuries. The most significant principle illustrated is the need for a coordinated, multidisciplinary approach that is focused on 
early aggressive resuscitation, rapid diagnosis, and emergent transfer to a cardiac surgery service. The second principle is that of a staged definitive repair. The decision to proceed with a stepwise repair was taken for several reasons. First, the significant amount of time on cardiopulmonary bypass, coupled with large transfusion and hypothermia, greatly increases the risk of morbidity and mortality. A single-stage procedure could have resulted in significant coagulopathy and possibly an increased risk of anastomotic breakdown. The primary goal of this procedure was to repair her LV and establish source control of the colonic disruption.

With regard to the cardiac repair, having a plan for peripheral cannulation was prudent. The peripheral cannulation and early cardiopulmonary bypass allow management of unforeseen complications such as free rupture and catastrophic hemorrhage. The LV was repaired in a similar fashion to a conventional ventricular rupture or chronic ventricular aneurysm. ${ }^{5,6}$ The rationale for using autologous pericardium to exclude the colonic defect was derived from the theory that it may be less prone to infection, given that it was in direct contact with the alimentary canal until definitive thoracic repair.

Delaying definitive thoracic surgical intervention has several benefits and challenges; the optimum timing for excision of the colonic interposition graft and esophageal diversion is unknown in this situation. Delayed repair does allow the patient to stabilize following the initial surgical intervention. However, there is no way to know how long it will take the patient to stabilize between operations, which may expose the patient to unanticipated complications. In an effort the save the conduit, debridement and repair of the colonic defect are attempted initially. Primary repair was not successful due to the significant contamination and subsequent tissue damage. Colonic conduit excision and esophageal diversion were ultimately required for patient safety. In conclusion, the authors would recommend definitive cardiac repair followed by staged esophageal resection and cervical esophagostomy with delayed definitive esophageal reconstruction.

\section{References}

1 Young MM, Deschamps C, Trastek VF, et al. Esophageal reconstruction for benign disease: early morbidity, mortality, and functional results. Ann Thorac Surg 2000;70(05):1651-1655

2 Thomas P, Fuentes P, Giudicelli R, Reboud E. Colon interposition for esophageal replacement: current indications and long-term function. Ann Thorac Surg 1997;64(03):757-764

3 Mansour KA, Bryan FC, Carlson GW. Bowel interposition for esophageal replacement: twenty-five-year experience. Ann Thorac Surg 1997;64(03):752-756

4 Pantelides ML, Fitzgerald MD. Left ventriculo-colic fistula-a late complication of colonic interposition for the oesophagus. Postgrad Med J 1988;64(755):710-712

5 Cooley DA. Ventricular endoaneurysmorrhaphy: a simplified repair for extensive postinfarction aneurysm. J Card Surg 1989; 4(03):200-205

6 Dor V, Saab M, Coste P, Kornaszewska M, Montiglio F. Left ventricular aneurysm: a new surgical approach. Thorac Cardiovasc Surg 1989;37(01):11-19 